\title{
Design and Simulation Evaluation of Distributed Driving Control for Combination of Personal Vehicles
}

\author{
Takeki Ogitsu* \\ Gunma University, 1-5-1, Tenjin-cho, Kiryu-shi Gunma 376-8515, Japan \\ *Corresponding Author: ogitsu@ gunma-u.ac.jp
}

\begin{abstract}
Recently, several vehicle manufacturers in Japan have unveiled the concept model of personal vehicles (PVs). PVs are small single- or double-seated electric vehicles and are specifically suitable for short-distance trips. Considering the trend of vehicle use in Japan, PVs are suitable for daily transportation. However, several PVs will be required to transport many bags or people, and each PV would require a driver. Thus, potential users of PV choose passenger vehicles, which have a higher load capacity than PVs. Therefore, this study aims to solve this issue by enabling a combination of PVs to be operated as a single vehicle, thus expanding the utility of PVs. A dynamic vehicle model using a neural network is developed and evaluated for accomplishing distributed driving control of the combination of PVs. The simulation evaluations validate the feasibility of the combination of PVs and clarify the relationship between driving distance and modeling accuracy.
\end{abstract}

Keywords: Personal Vehicle, Platooning, Machine Learning, Neural Network, Dynamic Modeling.

\section{Introduction}

Recently, the use of PVs has garnered interest in Japan. PVs are small single- or double-seated electric vehicles and are specifically suited for short-distance trips. Target users of PVs are those who seek transportation for distances that are too long for walking or cycling and too short for using motorbikes or passenger vehicles. In Japan, the average number of people in a passenger vehicle is about 1.3/unit and the average distance of a trip is about $10 \mathrm{~km}$. This statistic agrees with the above assumption regarding vehicle use in Japan.

PVs are expected to be used for pleasure or business trips within a region. In Japanese suburban areas, public transportation is inconvenient, and hence, visitors rent passenger vehicles or walk to their destinations. If they can rent PVs at a lower cost than passenger vehicles, they can conveniently explore the entire city instead of only visiting areas close to stations. Therefore, the use of PVs would lead to the revitalization of suburban cities. In addition, PVs can be customized depending on the characteristics of each city because they are cheaper than passenger vehicles.

Moreover, PVs are expected to be used for transportation from one's home or office, public transportation, and delivery services because they are faster and more comfortable than walking or cycling. PVs can be especially beneficial for delivery services because they can reduce operation costs, for example, fuel cost. In addition, PVs can enhance the brand image of a company because they reduce noise pollution and the environmental burden. Therefore, several Japanese municipalities are discussing the reconstitution of social transportation systems and are running trials of PVs within special zones.

However, PVs have a lower load capacity than passenger vehicles. This may be paradoxical. Although users of passenger vehicles need the performance of PVs, they choose passenger vehicles when they need to travel with many bags or when many people are traveling together even if this case is less frequent. In general, each PV requires one driver; therefore, many drivers are needed in the case of many bags or people. This limits the utility of PVs.

To solve this issue, this study proposes PVs that can be used in combination. Many important advantages of combination of vehicles have been reported ${ }^{(1-5)}$. We have been attempting to apply combination of vehicles to PVs as well as passenger vehicles and trucks. This paper mainly focuses on a mechanical method of combining PVs. Previous studies have developed and evaluated semi-mechanical and 
electronic methods of combining vehicles ${ }^{(6-10)}$.

If a combination of PVs can be operated as a single PV, the utility of PVs can be expanded. From the viewpoint of regulation, PVs combined by the mechanical method described in this study are easier to operate. However, a PV is assumed to be used as a single vehicle. Thus, improving the driving motor output of PVs to enable towing of other PVs when used in combination is not desirable. The combination of PVs should be under distributed driving control because this control can complement the output of a $\mathrm{PV}$. To operate combined PVs as a single PV, the driving control should be designed such that the vehicles' driving characteristics do not drastically fluctuate after the combination.

This study develops a dynamic vehicle model for accomplishing distributed driving control for a combination of PVs using neural networks and evaluates the model through simulations. Distributed driving control aims to maintain the driving characteristics of the combined vehicles at the same level as that of a single vehicle by sharing vehicle state quantities via $\mathrm{V} 2 \mathrm{~V}$ communication. The vehicle modeling can be performed dynamically on the assumption that users get on/off the vehicle and load/unload their baggage.

The rest of the paper is organized as follows. Section 2 describes the dynamic vehicle model using a neural network. Section 3 presents the conditions and results of the simulation evaluation to validate the dynamic vehicle model using a neural network. Finally, Section 4 concludes the paper.

\section{Simulation Modeling}

This section describes the system architecture of the dynamic model using neural networks for accomplishing distributed driving control of the combination of PVs.

\subsection{System Architecture}

As explained above, this study aims to solve technological issues of PVs to enable their operation in combination, and as a result, expand the utility of PVs. This study focuses on the mechanical method of combining PVs. When PVs are combined mechanically, they must be under distributed driving control to complement the output forces of the PVs. Moreover, the driving characteristics of the vehicles in combination must not drastically fluctuate in order to ensure operability of the driver of the leading vehicle.
In particular, mechanical energy must not be transferred via the coupling device to the other PVs in the combination. This also decreases the vibration generated by the vehicle combination, thus improving ride comfort.

Here, the leading vehicle is referred to as vehicle A and the following vehicle is referred to as vehicle B. Vehicle A uses its braking/driving characteristics when used alone as the standard and calculates the difference between the braking/driving characteristics of vehicles $\mathrm{A}$ and $\mathrm{B}$. The calculated difference is used for machine learning by the neural network and communicated to vehicle B's command value of braking/driving. In other words, when vehicles $\mathrm{A}$ and $\mathrm{B}$ are combined, the model calculates the command value of braking/driving to make the braking/driving characteristics of the vehicle $\mathrm{B}$ close to those of vehicle A operating alone. If more than three vehicles are combined, the middle vehicle should equip functions of vehicle $\mathrm{A}$ and B.

\subsection{Combination of Vehicles using Machine Learning}

A dynamic model for combining PVs is designed using the selective desensitization neural network (SDNN) in paper $^{(12)}$ as a reference. SDNN can perform learning in lesser time and has a better generalization ability than other neural networks. To evaluate the performance of SDNN, previous studies have developed and validated a dynamic model for combination of vehicles using monotonic acceleration ${ }^{(13-15)}$. This study expanded the dynamic model to combining PVs so that it can be employed in actual operation.

\section{Simulation Evaluation}

This section describes the conditions and results of the simulation evaluations of the dynamic model for combining PVs using the neural network described in Section 2.

\subsection{Evaluation Conditions}

Two combined vehicles are simulated on a flat straight road to evaluate the dynamic vehicle model for combining PVs using a neural network. The vehicle behavior model of the simulation only considers longitudinal control because this study assumes only driving on a straight road. The vehicle behavior model calculates vehicle acceleration, velocity, and position from the vehicle's weight, rotation resistance, air resistance, and the force generated from the tires. The force generated from the tires of each vehicle is calculated from the braking/driving characteristics map. The characteristics map is a matrix of the force generated from 
the vehicle, which consists of the command value and velocity. The mass of vehicle $B$ is 1.5 times that of vehicle $A$. The command value of vehicle $\mathrm{B}$ is calculated with the proposed system.

Under the above conditions, vehicle $\mathrm{A}$ is under proportional velocity control in order to simulate a typical PV driver's behavior of acceleration/deceleration. The target velocity of vehicle $A$ is set at $10 \mathrm{~km} / \mathrm{h}$ for $30 \mathrm{~s}$ after the beginning of the experiment and then set to $0 \mathrm{~km} / \mathrm{h}$. After driving for $55 \mathrm{~s}$, vehicle A repeats the driving behavior from the beginning of the experiment, thus maintaining the learning state of the neural networks.

\subsection{Evaluation Results}

Fig. 1 shows the velocity fluctuation of the combined vehicles for 10 cycles. The deeper color of the solid lines indicates later cycles. These results show that before learning, the combination of vehicles has insufficient output power for acceleration/deceleration compared with that of vehicle $\mathrm{A}$ as a single vehicle. However, in later cycles, the output power for acceleration/deceleration of the combination of vehicles is closer to that of vehicle $\mathrm{A}$ as a single vehicle. This indicates that by using the proposed system, the driving characteristics of the combination of vehicles can become almost the same as that of vehicle $\mathrm{A}$ as a single vehicle even if the mass of the vehicle following vehicle A significantly fluctuates because of the weight of baggage or people getting on/off the vehicle.

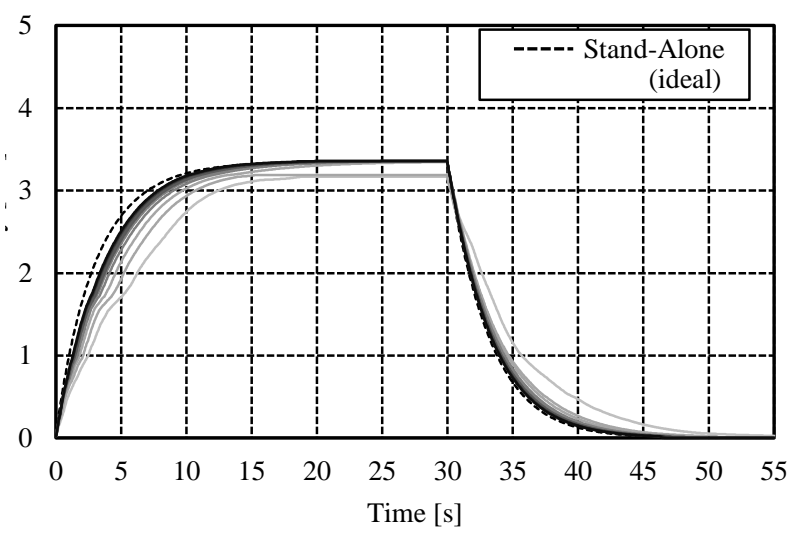

Fig. 2. Simulation Results.

\section{Conclusions}

A dynamic vehicle model was developed for mechanically combining PVs using SDNN, which can perform learning in lesser time and has a better generalization ability than other neural networks. Simulation evaluations were conducted to clarify the relationship among the learning performance, driving distance, and accuracy of the proposed model. The simulation results validated the accomplishment of distributed driving control for combination of PVs, which will enable expanding the utility of PVs.

In future work, the author will conduct demonstration experiments by installing the distributed driving control in actual PVs.

\section{References}

(1) J. Larson, K. Liang, and K. Johansson : “A Distributed Framework for Coordinated Heavy-duty Vehicle Platooning" Special Issue of IEEE Transactions on Intelligent Transportation Systems, 2014

(2) S. Joo, X. Lu, J. Hedrick : "Longitudinal Maneuver Design in Coordination Layer for Automated Highway System" Proceedings of the IEEE American Control Conference, pp. 42-47, 2003

(3) S. Tsugawa : "A History of Automated Highway Systems in Japan and Future Issues", The IEEE International Conference on Vehicular Electronics and Safety, pp. 2-3, 2008

(4) S. Tsugawa : "Results and Issues of An Automated Truck Platoon within The Energy ITS Project”, IEEE Intelligent Vehicles Symposium Proceedings, pp. 642647, 2014

(5) T. Ogitsu, T. Hirano, and M. Omae : "Design and Evaluation of Transitional Process of Platooning of Heavy-Duty Vehicles" Proceedings of the 18th World Congress on Intelligent Transport Systems, CD-ROM 2011

(6) T. Ogitsu, T. Ikegami, S. Kato, and H. Mizoguchi : "Development of Hard-Link Type Mobility for Small EV; Distributed Drive Control using State of Preceding Vehicle and Machine Learning" Proceedings of the Society of Automotive Engineers of Japan, No. 40-14, pp. 1-4, 2014

(7) T. Ogitsu, K. Tanaka, N. Matoba, S. Kato, and H. Mizoguchi : "A Study of Coupled Mobility Development and Evaluation of Semi-Mechanical Coupling Type Following System Using Small EV -" Transactions of the Society of Automotive Engineers of Japan, Vol. 45, No. 2, pp. 463-468, 2014

(8) M. Omae, T. Ogitsu, R. Fukuda, and W. Chang : "Longitudinal Control Algorithm for CACC 
(Cooperative Adaptive Cruise Control System) of Heavy-Duty Vehicles" Transactions of the Society of Automotive Engineers of Japan, Vol. 44, No. 6, pp. 1509-1515, 2013

(9) T. Ogitsu, M. Omae, and H. Shimizu : "A Study of Platoon Control System Using Moving Base RTK and Vehicle-to-Vehicle Communication Network" Transactions of the Society of Automotive Engineers of Japan, Vol. 43, No. 4, pp. 911-916, 2012

(10) M. Omae, T. Ogitsu, and H. Shimizu : "Soft-Link Control for Electric Light Vehicle," The World Electric Vehicle Association Journal, Vol. 2007, No. 1, pp. 32-37, 2007

(11)T. Ogitsu, T. Ikegami, S. Kato, and H. Mizoguchi : "Study of Coupling Technologies for Personal Vehicle Transit" Proceedings of the 3rd International Conference on Connected Vehicles and Expo, CD-ROM, 2014

(12) T. Shinbo, K. Yamane, F. Tanaka, and M. Morita : "Value Function Approximation in Reinforcement Learning Using Selective Desensitization Neural Networks" The IEICE Transactions, J93-D, 6, pp. 837847,2010

(13) T. Ogitsu, T. Ikegami, S. Kato, and H. Mizoguchi : "Neural Network On-Line Modeling for MechanicallyCoupled Vehicle", Proceedings of IEEE European Modelling Symposium 2014, CD-ROM, 2014

(14) T. Ogitsu, S. Kato, and H. Mizoguchi : "Distributed Driving System for Coupled Small EV Using Neural Network and Load Cell", Proceedings of IEEE 6th International Conference on Intelligent Systems, Modelling and Simulation, CD-ROM, 2015

(15) T. Ikegami, T. Ogitsu, and H. Mizoguchi : "Distributed Power Control Evaluation of Hard-Link-Type Mobility Using Velocity and Load Data”, Proceedings of IEEE International Conference on Connected Vehicles and Expo 2015, CD-ROM, 2015 